SLAC-TN-10-041

LCLS-TN-08-2

\title{
Magnetic Measurements of the Background field in the Undulator Hall
}

\author{
Andrew Fisher, Heinz-Dieter Nuhn, James Welch
}

$2 / 13 / 2008$

\begin{abstract}
The steel present in the construction of the undulator hall facility has the potential for changing the ambient fields present in the undulator hall. This note describes a measurement done to make a comparison between the fields in the hall and in the Magnetic Measurement Facility.
\end{abstract}

\section{1. Introduction ${ }^{1}$}

In order for the undulators to have the proper tuning, the background magnetic field in the Undulator Hall should agree with the background field in the Magnetic Measurements Facility within .5 gauss.

In order to verify that this was the case measurements were taken along the length of the undulator hall, and the point measurements were compared to the mean field which was measured on the MMF test bench.

\section{2. Test Setup}

The device used to measure the magnetic fields in the tunnel was a Barrington Instruments MAG-03 3 axis Fluxgate probe with a range of -5 to 5 gauss. The measurements were completed at an interval of 12 meters apart down the length of the undulator hall. The device was aligned so that the $\mathrm{Z}$ of the probe pointed appoximately in the direction of the beam, the $\mathrm{Y}$ direction at the cieling, and the X direction at the left wall of the tunnel. All measurements were taken at a height of $\sim 1.3$ meters off of the floor of the undulator hall. The $\mathrm{Z}$ position was fixed using a tape measure measurement from the beginning of the hall, and then with place holders every 4 meters.

\footnotetext{
${ }^{1}$ Work supported in part by the DOE Contract DE-AC02-76SF00515. This work was performed in support of the LCLS project at SLAC.
} 


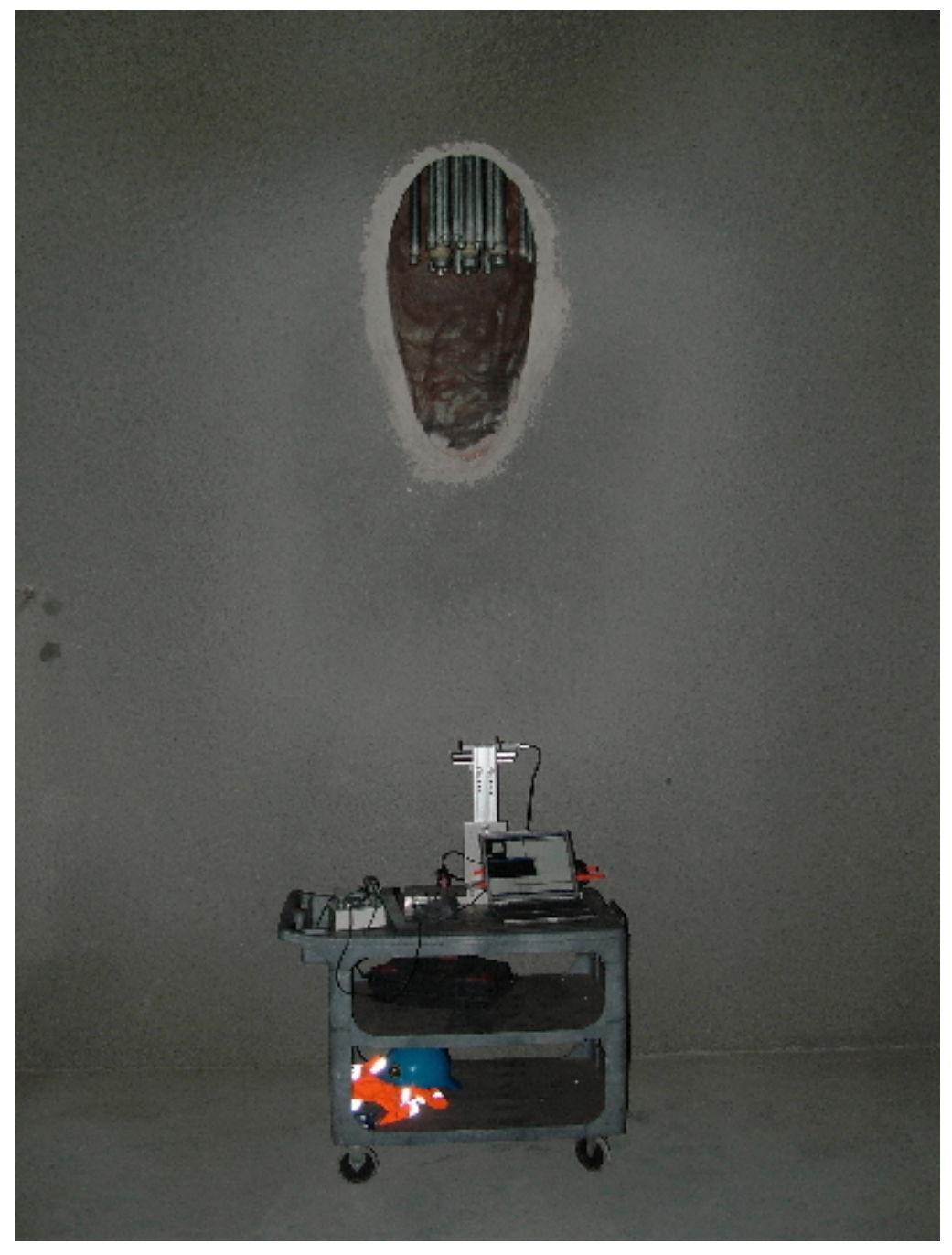




\section{3. Data And Conclusions}

After measuring the data in the tunnel, the data was plotted to get the field profile. Although there was a low field area at the beginning of the undulator hall, and an increase in the field approximately underneath the service building, overall the field agreed very closely with the earth's field without modification.

The data was also compared with the mean field on the MMF undulator stand as taken by Yurii Levashov. The data he took was divided into $\mathrm{X}$ and $\mathrm{Y}$ components and then compared to the $\mathrm{X}$ and $\mathrm{Y}$ components of the field in the Undulator Hall. The $\mathrm{Y}$ field had a maximum deviation from the table measurements of .21 gauss, and a mean deviation of .065 gauss, and the $\mathrm{X}$ field had a max of .16 gauss and a mean of .09 gauss.

\section{4. Plots}

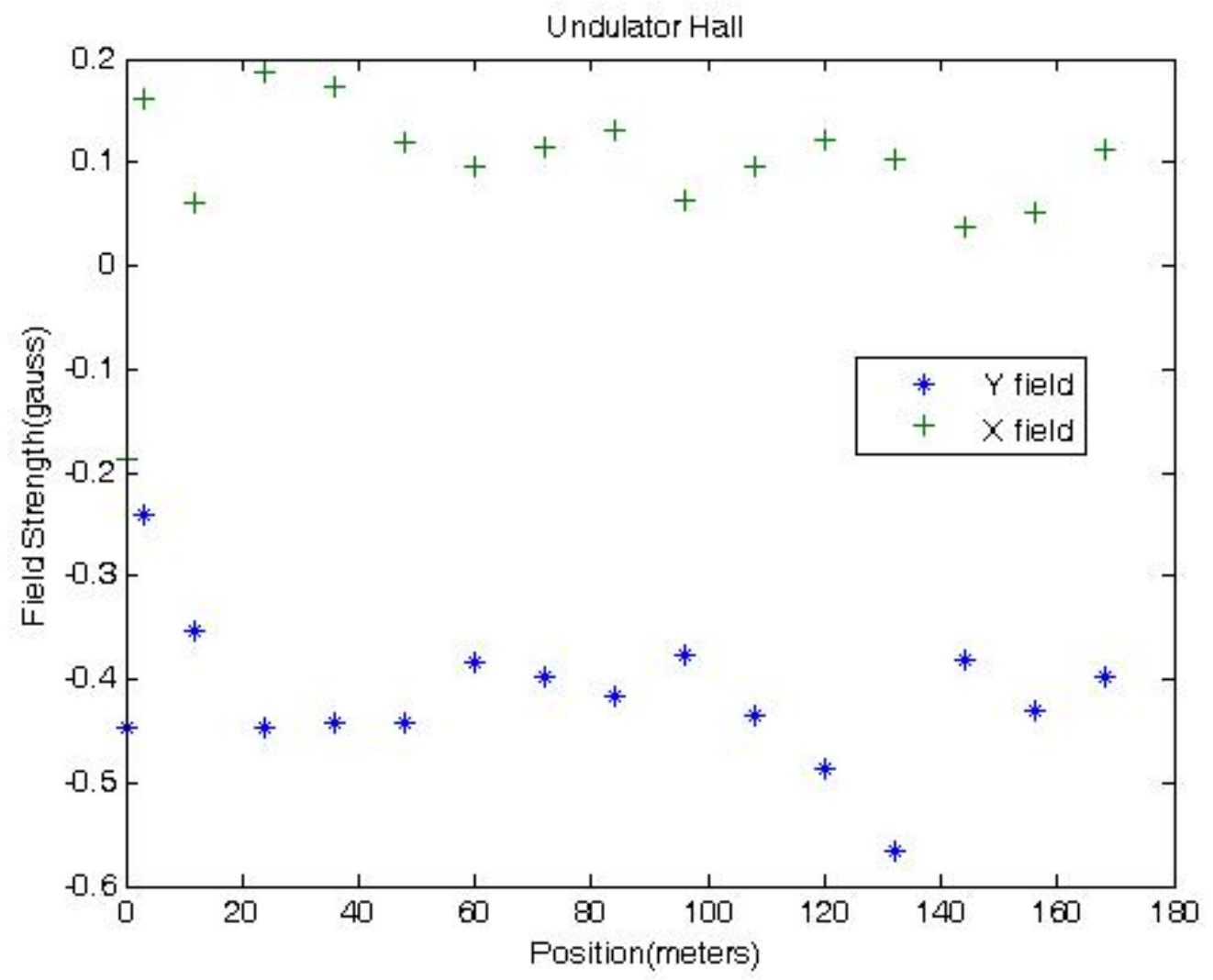




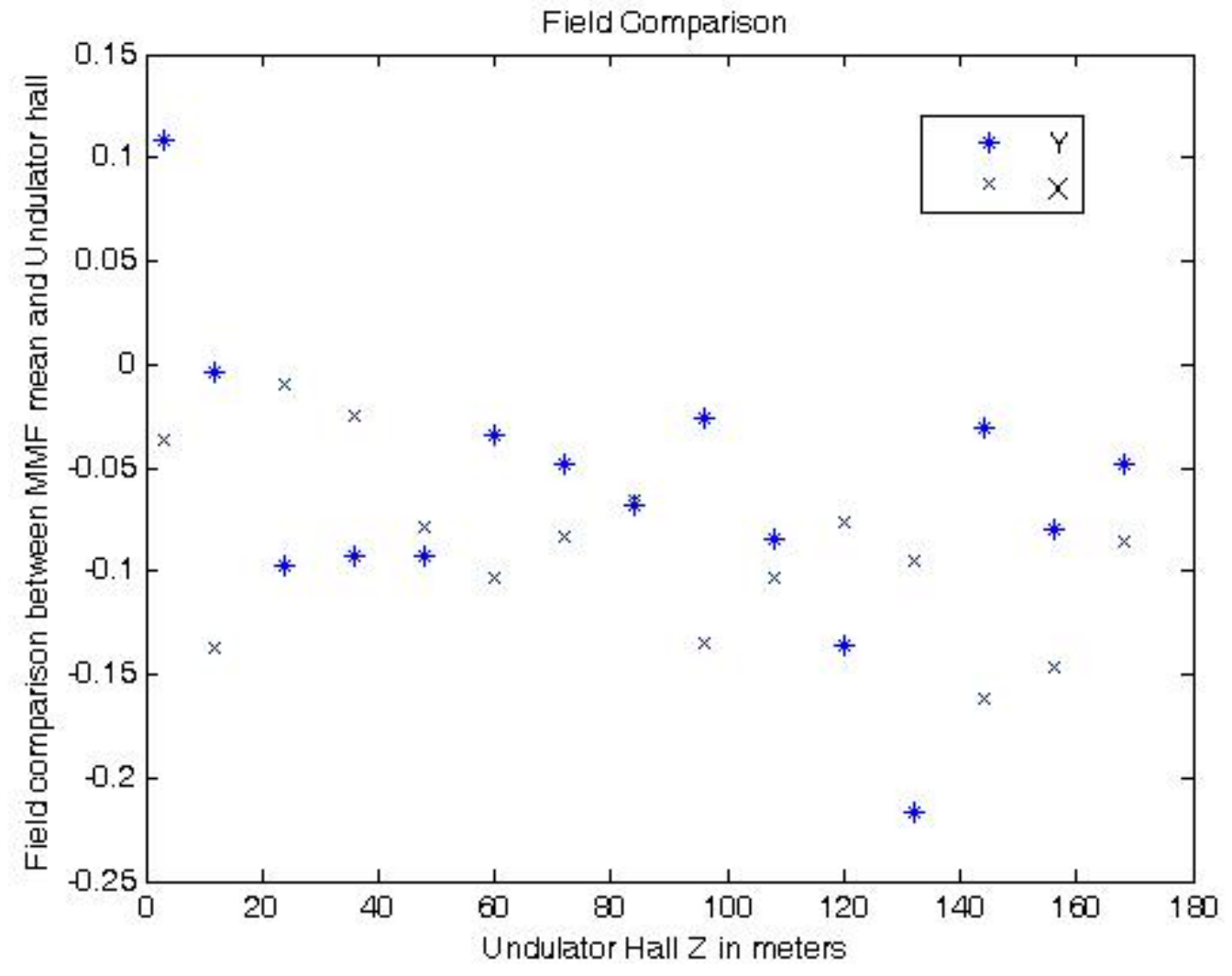

